\title{
NON-FERMI LIQUID BEHAVIOR OF CORRELATED FERMIONS IN APPLIED FIELD
}

\author{
P. Korbel, J. Spalek \\ Institute of Physics, Jagiellonian University, Reymonta 4, 30-059 Kraków, Poland \\ AND W. Wósclk \\ Institute of Physics, Technical University, Podchorążych 1, 30-084 Kraków, Poland
}

We have determined an instability of the Fermi-liquid state of almost localized fermions in an applied magnetic field. It is proposed that a transition to a strongly correlated fermions (statistical-spin-liquid) state takes place at that point. The resultant magnetization curve and the field dependence of the specific heat are calculated and compared with those for $\mathrm{CeRu}_{2} \mathrm{Si}_{2}$.

PACS numbers: 71.27.+a, 71.30.+h, 71.10.Fd

The metallic state close to Mott-IIubbard localization is commonly regarded as a Fermi-liquid state of correlated fermions [1]. The quasiparticles in this liquid have spin-dependent effective masses (if the band filling $n \neq 1$ ), and experience a nonlinear molecular field in the spin polarized state [2]. The principal question is: what happens for $n \neq 1$ if we apply a magnetic field and the number of double occupancy $d^{2} \longrightarrow 0$, i.e. magnetization $m \equiv n_{\uparrow}-n_{\downarrow} \longrightarrow n$ ? Does it transform gradually into a gas of fermions with one spin direction up or a non-Fermi liquid state comes into play before the system saturates magnetically?

The purpose of this paper is to demonstrate that at the point of instability of the almost localized Fermi liquid (ALFL) a transition to the spin liquid state takes place. Consequently, we calculate the magnetization curve exhibiting a mixed itinerant-localized behavior, as well as the applied field dependence of the linear specific heat coefficient $\gamma \equiv C / T$ across the transition. In Fig. 1a we have displayed the field dependence of the double occupancy $d^{2} \equiv\left\langle n_{i \uparrow} n_{i \downarrow}\right\rangle$ in the narrow band of correlated fermions, for three different relative temperatures $t \equiv k_{\mathrm{B}} T / W$ (W is the bare band width, $U / U_{\mathrm{c}}$ is the relative magnitude of interaction, $U_{\mathrm{c}}=2 W$ and $n$ is the band filling). At the points of instability (marked by the dashed lines) there is a metamagnetic transition, associated with a jump in $d^{2}$ and magnetization $m$. For the fields $h \equiv \mu_{\mathrm{B}} H_{\mathrm{a}} / W$ beyond this point $\left(h=h_{\mathrm{c}}\right)$ we obtain a nonphysical values of $d^{2}<0$, so the ALFL state is unstable. On the physical grounds one can visualize the state $d^{2}=0$ as a liquid of itinerant spins, since the hopping takes place only via empty lattice sites. Therefore, we proposed [3] that such a state 


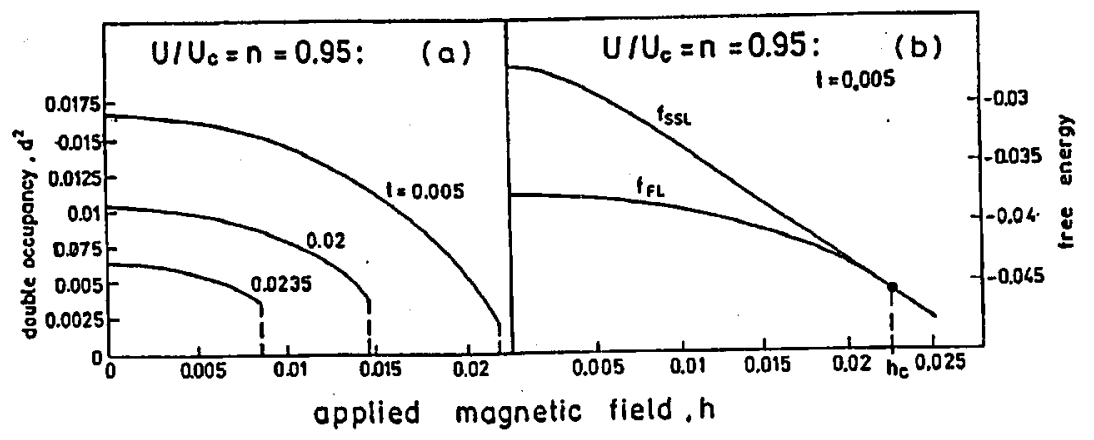

Fig. 1. (a) Field variation of the double occupancy; (b) the free energies of ALFL and SSL states ( $f_{\mathrm{FL}}$ and $f_{\mathrm{SSL}}$, respectively) as a function of applied field $h$.

is described by the particles with bare band energies, but with the singlet spin configuration excluded in reciprocal $k$ space.

The statistical properties of such a spin liquid (SSL) in an applied field are governed by the distribution $\left\langle n_{k}\right\rangle=1 /\left\{1+a \exp \left[\beta\left(\epsilon_{k}-\mu\right)\right]\right\}$, with $a=$ $1 /\left[2 \cosh \left(\beta \mu_{\mathrm{B}} H_{\mathrm{a}}\right)\right]$, where $\mu$ is the chemical potential and $\beta=1 / k_{\mathrm{B}} T$. The magnetic moment (per site) $m$ changes with the field according to $m=\tanh \left(\beta \mu_{\mathrm{B}} H_{\mathrm{a}}\right)$, i.e. it has the same shape as in the localized-moment case. Finally, for a constant value $(1 / W)$ of the density of states in the band interval $[-W / 2, W / 2]$, the free energy (per atom) of the liquid has the form

$$
f_{\mathrm{SSL}}=\frac{F_{\mathrm{S}}}{W}=-\frac{1}{2} n(1-n)-\frac{\pi^{2}}{6} t^{2}-n t \ln [2 \cosh (h / t)] .
$$

For $n=1$ and $H_{\mathrm{a}}=0$ this expression reduces to $(-\ell \ln 2)$ for free spins (this means that we do not include the exchange interactions for the sake of simplicity of the discussion). In Fig. 1b we display the free energies of the ALFL (as presented by $f_{\mathrm{FL}} \equiv F_{\mathrm{FL}} / W$ and SSL states for $n=U / U_{\mathrm{c}}=0.95$ and $t=5 \times 10^{-3}$. The two energies coincide exactly at the point $H_{\mathrm{c}}$, at which $d^{2} \equiv 0$ for $T=0$. This means that the two states coexist at $H_{\mathrm{a}}=H_{\mathrm{c}}$, and that SSL is stable for $h>h_{\mathrm{c}}$. In other words, at $h_{\mathrm{c}}$ the Fermi liquid transforms into a gas of hopping spins with an unrenormalized mass but with a changed statistics.

The resultant magnetization curve with a jump in the regime of ALFL $\longrightarrow$ SSL transition is shown in Fig. 2 for the parameter $n=U / U_{c}=0.95$ (the inset provides the temperature variation of the critical field $h_{\mathrm{c}}$ ). For $n \geq 0.973$ the curve will exhibit only an upward turn followed by an approach to saturation, without a subsequent jump; this metamagnetic behavior combines the Fermi-liquid (for $h<h_{\mathrm{c}}$ ) and localized moment-behavior (for $h>h_{\mathrm{c}}$ ). The same type of behavior is reflected also in the linear specific heat coefficient $\gamma \equiv C / T$. The discontinuous transition is accompanied by the jump in $\gamma$; the crossover behavior for $n=0.977$ is displayed in Fig. 3 . The curves and the inset illustrate a general trend, which is characterized by a strong band-filling dependence of the physical properties when $\delta \equiv 1-n \ll 1$. So the almost localized fermions appear indeed close to the Mott localization, i.e. for $\delta \ll 1, U / U_{\mathrm{c}}-1 \ll 1$. The coefficient $\gamma$ is proportional to the effective mass $m^{*}$, which in the ALFL case is spin dependent, 


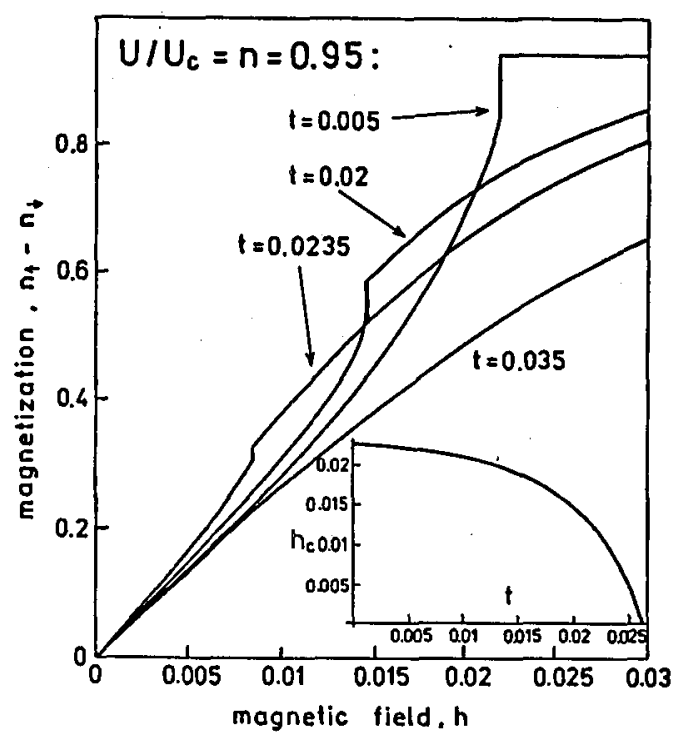

Fig. 2. The magnetization curve in the region of ALFL $\rightarrow$ SSL transition; the inset shows the temperature dependence of the critical field for the transition to take place.

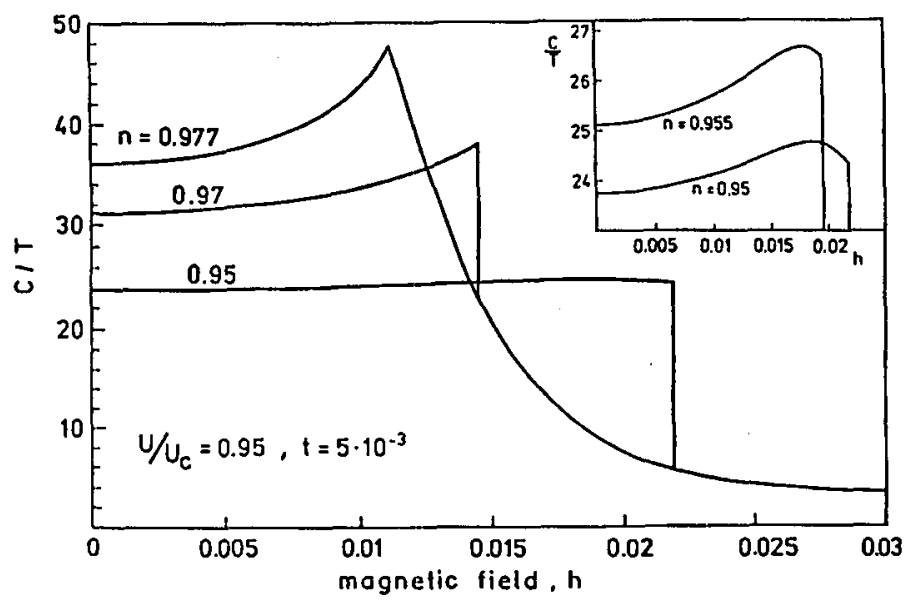

Fig. 3. Field dependence of the linear specific heat coefficient (also in the inset). The cusp or discontinuity signals the crossover behavior.

i.e. $m^{*} / m_{0}=1 / q_{\uparrow}+1 / q_{\downarrow}$. Above the transition only one mass with the value close to the band theory value $m_{0}$ should be detected. This and other properties displayed in Figs. 2 and 3 seem to reflect the observed behavior for heavy-fermion systems such as $\mathrm{CeRu}_{2} \mathrm{Si}_{2}$ [4].

In summary, we have introduced a physical model of the transformation of a Fermi liquid into a non-Fermi (spin) liquid in the magnetic field. This mean-field 
approach should provide a realistic treatment of the ALFL instability, since the spin-fluctuation effect are suppressed by rather a strong magnetic field.

The paper was supported by the Committee for Scientific Research through grant No. 2P302 09305.

\section{References}

[1] For recent reviev see: J. Spałek, W. Wójcik, in: Spectroscopy of the Mott Insulators and Correlated Metals, Eds. A. Fujimori, Y. Tokura, in series Springer Tracts in Solid State Science, Vol. 119, Springer, Berlin 1995, p. 41.

[2] J. Spałek, W. Wójcik, P. Korbel, Acta Phys. Pol. A 92, (1997).

[3] J. Spałek, W. Wójcik, Phys. Rev. B 37, 1532 (1988); K. Byczuk, J. Spałek, Phys. Rev. $B$ 50, 11403 (1994).

[4] P. Haen, J. Flouquet, F. Lapierre, P. Lejay, G. Remenyi, J. Low Temp. Phys. 67, 391 (1987); C. Paulsen, A. Lacerda, L. Puech, P. Haen, P. Lejay, J.L. Tholence, J. Flouquet, A. de Visser, ibid. 81, 317 (1990); H. Aoki, S. Uji, A.K. Albessard, Y. Onuki, Phys. Rev. Lett. 71, 2110 (1993). 